\title{
Desempenho Reprodutivo de Cabras Alpinas Tratadas com hCG Cinco Dias Após o Acasalamento 1
}

\author{
Jeferson Ferreira da Fonseca ${ }^{2}$, Ciro Alexandre Alves Torres $^{3}$, Vitor Valério Maffili ${ }^{4}$, Alan Maia \\ Borges $^{5}$, Cláudio José Borela Espeschit ${ }^{6}$, Paula de Zorzi Balbinot ${ }^{7}$, Rita Flávia Miranda Oliveira ${ }^{8}$, \\ Pedro A. G. Leite ${ }^{9}$
}

\begin{abstract}
RESUMO - Objetivou-se com este estudo avaliar o efeito da administração de hCG sobre o desempenho reprodutivo de cabras Alpinas durante a estação de acasalamento natural. Trinta e duas fêmeas nulíparas e 124 lactantes, após a identificação de estro e acasalamento, foram aleatoriamente divididas, de acordo com a categoria, em dois tratamentos. Em T1 (n=75) e T2 ( $\mathrm{n}=81)$, os animais receberam $1 \mathrm{~mL}$ de solução salina ou 250 UI de hCG, respectivamente, por via intramuscular cinco dias após o acasalamento. A gestação foi verificada por ultra-sonografia transabdominal (probe de 3,5 MHz) nos dias 35 e 70 após o acasalamento, para detecção e confirmação da gestação, respectivamente. As taxas de gestação não diferiram entre T1 (86,7\%) e T2 (70,6\%) para nulíparas e T1 (78,3 \%) e T2 (84,4 \%) para lactantes. Não houve diferença entre a taxa de parição (75,0 e 75,7\%), o período de gestação (150,47 e 150,80 dias) e a prolificidade (1,75 e 1,80 fetos) entre os animais do T1 e T2, respectivamente. A prolificidade foi superior em cabras lactantes $(1,90)$ que em nulíparas $(1,41)$. A administração de hCG cinco dias após o acasalamento não elevou o desempenho reprodutivo em cabras da raça Alpina.
\end{abstract}

Palavras-chave: caprino, hCG, desempenho reprodutivo

\section{Reproductive Performance of Alpine Goats Treated with hCG Five Days After Breeding}

ABSTRACT - The effect of hCG administration on reproductive performance of Alpine goats during the natural breeding season was evaluated. Thirty-two nulliparous and 124 lactating goats, after estrus identification and breeding, were randomly assigned according to the categories to two treatments. In T1 $(n=75)$ and T2 $(n=81)$ the animals received $1 \mathrm{~mL}$ of saline solution or $250 \mathrm{IU}$ of hCG intramuscularly, respectively, five days after breeding. Pregnancy was detected and confirmed on days 35 and 70 after breeding by transabdominal ultrasonography (3.5 MHz probe), respectively. Pregnancy rate did not differ between T1 (86.7 \%) and T2 (70.6\%) for nulliparous and T1 $(78.3 \%)$ and T2 $(84.4 \%)$ for lactating does. There were no differences for kidding rate $(75.0$ and $75.7 \%)$, gestation period (150.47 and 150.80 days) and prolificacy (1.75 and 1.80 fetuses) between T1 and T2, respectively. Prolificacy was superior in lactating (1.90) than in nulliparous (1.41) goats. The administration of hCG five days after breeding did not increase reproductive performance in Alpine goats.

Key Words: goat, hCG, reproductive performance

\section{Introdução}

O sucesso de qualquer sistema de produção animal depende principalmente do desempenho reprodutivo do rebanho. Para produção de carne, leite ou outros produtos de origem animal, é necessário que os animais se reproduzam eficientemente. Isto significa que os machos devem produzir gametas férteis e serem capazes de copular adequadamente. As fêmeas devem apresentar estro detectável e com produção de gametas férteis e serem acasaladas ou inseminadas em momento ideal, considerando-se a vida média dos

\footnotetext{
1 Parte da tese de doutorado do primeiro autor, financiada parcialmente pelo CNPq e pela FAPEMIG.

2 Médico Veterinário, DS, Pesquisador Embrapa Caprinos, CP D10, CEP: 62011-970, Sobral, Ceará, Brasil (jeferson@cnpc.embrapa.br).

${ }^{3}$ Médico Veterinário, PhD, Professor Titular, Departamento de Zootecnia, Universidade Federal de Viçosa, Av. P H Rolfs s/n, CEP: 36571-000, Viçosa, Minas Gerais, Brasil (ctorres@ufv.br).

${ }^{4}$ Médico Veterinário, MS, Departamento de Zootecnia, Universidade Federal de Viçosa, Av. P H Rolfs s/n, CEP: 36571-000, Viçosa, Minas Gerais, Brasil (vmaffili@hotmail.com).

${ }^{5}$ Médico Veterinário, DS, Departamento de Zootecnia, Universidade Federal de Viçosa, Av. P H Rolfs s/n, CEP: 36571-000, Viçosa, Minas Gerais, Brasil (alanmborges@hotmail.com).

${ }^{6}$ Médico Veterinário, DS, Técnico de Nível Superior, Departamento de Zootecnia, Universidade Federal de Viçosa, Av. P H Rolfs s/n, CEP: 36571-000, Viçosa, Minas Gerais, Brasil (espeschi@ufv.br).

${ }^{7}$ Médica Veterinária, Mestranda, Departamento de Veterinária, Universidade Federal de Viçosa, Av. P H Rolfs s/n, CEP: 36571-000, Viçosa, Minas Gerais, Brasil (paulabalbinot@yahoo.com.br).

${ }^{8}$ Zootecnista, DS, Professora Adjunto, Departamento de Zootecnia, Universidade Federal de Viçosa, Av. P H Rolfs s/n, CEP: 36571-000, Viçosa, Minas Gerais, Brasil (flavia@ufv.br).

${ }^{9}$ Médico Veterinário, Mestrando, Departamento de Zootecnia, Universidade Federal de Viçosa, Av. P H Rolfs s/n, CEP: 36571-000, Viçosa, Minas Gerais, Brasil (pedroleite@alunos.ufv.br).
} 
gametas masculino e feminino, para que ocorra uma fertilização normal. Seqüencialmente, o embrião deve encontrar um ambiente materno cronológica e fisiologicamente adequado ao seu desenvolvimento e nascimento a termo. Durante todo esse período, podem ocorrer perdas que culminam em inadequada taxa de nascimento, comprometendo todo o processo produtivo. Uma das principais razões dessas perdas é a mortalidade embrionária.

Muitos fatores, tanto maternais como embrionários têm sido apresentados para a perda embrionária, principal causa de falhas reprodutivas em mamíferos (Wilmut et al., 1986; Thatcher et al., 1994a,b). Existem várias formas de se prevenir a morte embrionária em bovinos, mas a maioria dos estudos está embasada em estratégias antiluteolíticas. A prevenção da luteólise ou o prolongamento da fase luteal por meio da administração de agentes luteotróficos pode aumentar as taxas de gestação (Thatcher et al., 2001). Em novilhas, a administração de gonadotrofina coriônica humana (hCG), potente agente luteotrófico, no quinto dia do ciclo estral, eleva as concentrações plasmáticas de progesterona (Fonseca et al., 2001a). Altas concentrações de progesterona reduzem a produção de prostaglandina- $\mathrm{F}_{2 \alpha}\left(\mathrm{PGF}_{2 \alpha}\right)$ pelo útero (Mann et al., 1995), favorecendo o estabelecimento da gestação tanto em bovinos (Rajamahendran \& Sianangama, 1992) quanto em ovinos (Kittok et al., 1983). Entretanto, caprinos são animais que dependem da progesterona luteal durante toda a gestação (Meites et al., 1951) e são freqüentemente acometidos de falha luteal (Sangha et al., 2002) e ainda carecem de maiores estudos sobre o efeito de agentes luteotróficos sobre a atividade luteal e, conseqüentemente, sobre sua eficiência reprodutiva.

Com base na dinâmica folicular ovariana descrita na espécie caprina (Ginther \& Kot, 1994; Castro et al., 1999), em que a dominância folicular efetiva ocorre somente na primeira e última ondas foliculares, a administração de hCG no quinto dia poderia promover a formação de corpo lúteo acessório semelhante ao que ocorre em bovinos e ovinos. Por outro lado, se o objetivo for estimular o desenvolvimento do corpo lúteo original do ciclo estral, a intervenção hormonal deve ser feita antes do $6^{\circ}$ dia $($ dia do estro $=$ dia 0$)$, visto que o corpo lúteo caprino atinge diâmetro máximo entre o $6^{\circ}$ e $9^{\circ}$ dia do ciclo estral (Jablonka-Shariff et al., 1993).

O objetivo neste estudo foi avaliar o efeito da administração de hCG cinco dias após o acasalamento sobre o desempenho reprodutivo de cabras Alpinas durante a estação de acasalamento natural.

\section{Material e Métodos}

O estudo foi conduzido de maio a novembro de 2002 no Setor de Caprinocultura do Departamento de Zootecnia da Universidade Federal de Viçosa (UFV), em Viçosa, na Zona da Mata de Minas Gerais, a $20^{\circ} 45^{\prime}$ 'S de latitude e $42^{\circ} 51^{\prime}$ 'WG de longitude, altitude média de 692,73 m, clima CWA pela classificação de Köppen (inverno seco e verão úmido), com temperatura média anual de $20,9^{\circ} \mathrm{C}$ e precipitação pluviométrica anual de $1.203 \mathrm{~mm}^{3}$.

Foram utilizadas 32 fêmeas nulíparas (idade superior a um ano) e 124 lactantes. (1,5 e 6 anos) entre a segunda e quarta lactações (lactação superior a 70 dias). Os animais foram mantidos em baias, suspensas ou não e alimentados ad libitum com silagem de milho e concentrado duas vezes ao dia. Água e minerais estiveram disponíveis permanentemente. A condição de escore corporal (CEC, variação de 1 a 5), avaliada por palpação da região lombar e esternal no dia do estro, foi de $3,7 \pm 0,5$ nas nulíparas e $3,2 \pm 0,8$ nas lactantes. O estro foi detectado duas vezes por dia, pela manhã e à tarde, por meio de macho cirurgicamente preparado (rufião por translocação peniana). Os sinais de estro observados foram: procura pelo macho, inquietação, vocalização, micção freqüente, batimento de cauda, contração, hiperemia e edema de vulva, descarga vaginal de muco e imobilidade à monta, sendo esta considerada como início da fase passiva do estro. As fêmeas foram acasaladas no momento da primeira aceitação e 12 horas após, caso permanecessem com o reflexo de imobilidade à monta. Foram utilizados quatro machos maduros e de fertilidade comprovada, avaliados semanalmente por meio de exame clínico e andrológico. Após detecção do estro (dia $0=$ dia do estro) e cobertura, os animais foram aleatoriamente distribuídos em dois tratamentos (T1 e T2), de acordo com as categorias (nulíparas e lactantes). Em T1 $(n=75)$ e T2 $(n=81)$, os animais receberam $1 \mathrm{~mL}$ de solução salina (controle) ou 250 UI (unidades interna- 
cionais) de $\mathrm{hCG}^{1}$, respectivamente, por via intramuscular, cinco dias após o acasalamento. O estudo compreendeu apenas um ciclo estral (primeiro serviço) e as fêmeas foram acasaladas de maio a julho.

Todas as fêmeas foram avaliadas por ultrasonografia transabdominal, com auxílio de probe de 3,5 $\mathrm{MHz}^{2}$ decorridos 35 e 70 dias da cobertura, para detecção e confirmação da gestação, respectivamente.

Após o nascimento, avaliou-se a taxa de parição, o período de gestação (dias) e a prolificidade.

A análise estatística compreendeu a análise de variância, para comprovação de diferenças entre período médio de gestação (dias) e prolificidade por fêmea existentes entre os tratamentos, cujas médias foram testadas pelo teste de SNK (5\%) e processadas pelo SAEG - Sistema para Análises Estatísticas (Ribeiro Jr., 2001). As taxas de gestação e parição foram avaliadas pelo teste do qui-quadrado (Ayres et al., 2000).

\section{Resultados e Discussão}

Não há relatos científicos sobre a administração de hCG cinco dias após o acasalamento em caprinos com o objetivo de elevar taxas de gestação. As taxas de gestação não diferiram $(\mathrm{P}>0,05)$ entre os animais do tratamento controle e os tratados com hCG (Tabela 1). Não houve perda fetal entre os dias 35 e 70 de gestação. Estudos em outras espécies de ruminantes domésticos, utilizando administração de hCG, têm apresentado resultados conflitantes. Em ovelhas em lactação, cujo estro foi induzido com progestágeno, Kittok et al. (1983) administraram 100 UI de hCG nos dias 11, 12 e 13 após o acasalamento e observaram aumento significativo na taxa de gestação do grupo tratado com hCG $(58 \%)$ quando comparado com o controle $(29 \%)$. Estes autores também observaram aumentos na concentração plasmática de P4 medida nos dias 12, 14 e 16 após o acasalamento, todavia a taxa de gestação média deste estudo foi relativamente alta (80\%). Nesta condição, mesmo que a hCG tenha promovido incrementos na concentração plasmática de progesterona, não repercutiria em elevação na taxa de gestação. De fato, segundo Staples \& Hansel (1961), para que uma gestação se estabeleça, há a necessidade de uma concentração limiar de progesterona. A partir deste nível, incrementos poste- riores não são capazes de elevar a taxa de gestação.

Nephew et al. (1994), após administrarem 100 UI de hCG 11,5 dias pós-acasalamento, também verificaram aumento na concentração plasmática de P4. Os mesmos autores, comparando os grupos tratados com hCG e controle (controle), reportaram aumentos significativos no diâmetro médio de blastocistos $(3,5 \pm 1,6$ vs $0,8 \pm 0,5 \mathrm{~cm})$, no peso luteal ( $616 \pm 15$ vs $433 \pm 24 \mathrm{mg}$ ), de proteínas ( $0,8 \mathrm{vs} 0,52 \mathrm{mg}$ ) e interferon-tau (IFN-tau) em lavados uterinos (10,7 vs $2,1 \mathrm{mg}$ ), sugerindo uma tendência $(\mathrm{P}<0,10)$ de elevação na taxa de gestação (91 vs $80 \%$ ) nos animais tratados. Os autores sugeriram que a hCG poderia estimular a progressão normal das secreções uterinas, levando a efeitos embriotróficos (aumento de tamanho), que repercutiriam em elevação da produção de IFN-tau, o que, conseqüentemente, antagonizaria o mecanismo luteolítico, potencializando o estabelecimento e a manutenção da gestação. Em vacas em lactação, Sianangama \& Rajamahendran (1992) administraram 1000 UI de hCG por via intramuscular no sétimo dia do ciclo estral e obtiveram elevação significativa na taxa de gestação de $40 \%$ e $66 \%$ para o grupo controle e o tratado com hCG, respectivamente. Por outro lado, o uso de hCG não resultou em elevação na taxa de gestação em outros estudos com bovinos (Greve \& LehnJensen, 1982; Schmitt et al., 1996; Fonseca et al., 2001b), semelhante aos resultados deste estudo, em caprinos.

A taxa de parição não diferiu $(\mathrm{P}>0,05)$ entre os tratamentos ou categoria animal (Tabela 2). De acordo com os resultados deste estudo (Tabela 2), 25\% dos animais gestantes interromperam suas gestações a partir de 70 dias de gestação (última avaliação ultrasonográfica). Há relativamente poucos estudos que relatam este fenômeno em caprinos. Segundo Engeland et al. (1998), 11\% dos animais abortam, parem fetos mortos ou estão vazios após terem sido considerados gestantes. Em um outro estudo, Engeland et al. (1997) observaram maiores perdas em animais com idade superior a sete anos (33\%), repetidores de estro (33\%), com número de fetos igual ou superior a três $(28 \%)$ e que já haviam tido perdas fetais (40\%). Esses dois estudos foram realizados em regiões de clima frio (Noruega). Em ambientes tropicais, onde os efeitos deletérios de altas temperaturas e umidade

\footnotetext{
${ }^{1}$ Vetecor ${ }^{\circledR}$, Laboratórios Calier do Brasil Ltda.

${ }^{2}$ Aloka, modelo SSD - 500, Tokyo, Japan.
} 
Tabela 1 - Taxa de gestação ao primeiro serviço (\%) em fêmeas caprinas tratadas com solução salina (controle) ou 250 UI de hCG cinco dias após o acasalamento

Table 1 - First service pregnancy rate (\%) in goats treated with saline (control) or 250 IU of hCG five days after breeding

\begin{tabular}{lcccc}
\hline $\begin{array}{l}\text { Categoria } \\
\text { Category }\end{array}$ & $\begin{array}{c}\text { Animais }(\mathrm{n}) \\
\text { Animals }\end{array}$ & $\begin{array}{c}\text { Controle }(\%) \\
\text { Control }\end{array}$ & $\begin{array}{c}\text { hCG }(\%) \\
h C G\end{array}$ & $\begin{array}{c}\text { Total }(\%) \\
\text { Total }\end{array}$ \\
\hline $\begin{array}{l}\text { Nulíparas }(\mathrm{n}) \\
\begin{array}{l}\text { Nulliparous } \\
\text { Lactantes }(\mathrm{n})\end{array}\end{array}$ & 32 & $86,7(13 / 15)$ & $70,6(12 / 17)$ & $78,1(25 / 32)$ \\
Lactating & 124 & $78,3(47 / 60)$ & $84,4(54 / 64)$ & $81,5(101 / 124)$ \\
\hline $\begin{array}{l}\text { Total }(\mathrm{n}) \\
\text { Total }\end{array}$ & 156 & $80,0(60 / 75)$ & $81,5(66 / 81)$ & $80,8(126 / 156)$ \\
\hline
\end{tabular}

Tabela 2 - Taxa de parição (\%) de fêmeas caprinas tratadas com solução salina (controle) ou 250 UI de hCG cinco dias após o acasalamento

Table 2 - Kidding rate (\%) of goats treated with saline (control) or 250 IU of hCG five days after breeding

\begin{tabular}{lcccc}
\hline $\begin{array}{l}\text { Categoria } \\
\text { Category }\end{array}$ & $\begin{array}{c}\text { Animais }(\mathrm{n}) \\
\text { Animals }\end{array}$ & $\begin{array}{c}\text { Controle }(\%) \\
\text { Control }\end{array}$ & $\begin{array}{c}\text { hCG }(\%) \\
h C G\end{array}$ & $\begin{array}{c}\text { Total }(\%) \\
\text { Total }\end{array}$ \\
\hline $\begin{array}{l}\text { Nulíparas }(\mathrm{n}) \\
\begin{array}{l}\text { Nulliparous } \\
\text { Lactantes }(\mathrm{n})\end{array}\end{array}$ & 25 & $92,3(12 / 13)$ & $83,3(10 / 12)$ & $78,0(22 / 25)$ \\
Lactating & 101 & $70,2(33 / 47)$ & $74,1(40 / 54)$ & $72,3(73 / 101)$ \\
\hline $\begin{array}{l}\text { Total }(\mathrm{n}) \\
\text { Total }\end{array}$ & 123 & $75,0(45 / 60)$ & $75,7(50 / 66)$ & $75,4(95 / 126)$ \\
\hline
\end{tabular}

sobre a reprodução são bem conhecidos (Wolfenson et al., 2000), a perda embrionária e/ou fetal poderia ser ainda maior. Como os acasalamentos foram feitos em maio a julho e a perda fetal ocorreu após 70 dias de gestação, é possível que temperatura e umidade elevada dos meses de outubro a novembro tenham tido efeito deletério sobre a gestação. Vários agentes infecciosos também podem estar envolvidos (Engeland et al., 1998). Todavia, no presente estudo, não foram identificadas as causas da perda fetal reportada.

Em ovinos, a administração de hCG após a inseminação estimulou a função luteal, repercutindo em aumento na concentração plasmática de P4. Todavia, não houve alteração nas taxas de gestação e de parição e na prolificidade (Ishida et al., 1999). Da mesma forma, neste estudo, tanto as taxas de gestação e parição quanto a prolificidade (Tabela 3) não foram significativamente alteradas pela administração de hCG cinco dias após o acasalamento.

O período de gestação (Tabela 4) não diferiu $(\mathrm{P}>0,05)$ entre os animais dos tratamentos. O período
Tabela 3 - Prolificidade de fêmeas caprinas tratadas com solução salina (controle) ou 250 UI de hCG cinco dias após o acasalamento

Table 3 - Prolificacy of goats treated with saline (control) or 250 IU of hCG five days after breeding

\begin{tabular}{|c|c|c|c|}
\hline $\begin{array}{l}\text { Categoria } \\
\text { Category }\end{array}$ & $\begin{array}{c}\text { Controle }(\mathrm{n}) \\
\text { Control }\end{array}$ & $\begin{array}{c}\mathrm{hCG}(\mathrm{n}) \\
\quad h C G\end{array}$ & $\begin{array}{c}\text { Total }(\mathrm{n}) \\
\text { Total }\end{array}$ \\
\hline $\begin{array}{l}\text { Nulíparas } \\
\text { Nulliparous }\end{array}$ & $1,33 \pm 0,49$ & $1,50 \pm 0,53$ & $1,41 \pm 0,50^{b}$ \\
\hline $\begin{array}{l}\text { Lactantes } \\
\text { Lactating }\end{array}$ & $1,91 \pm 0,53$ & $1,89 \pm 0,62$ & $1,90 \pm 0,58^{a}$ \\
\hline $\begin{array}{l}\text { Total } \\
\text { Total }\end{array}$ & $1,75 \pm 0,60$ & $1,80 \pm 0,61$ & $1,77 \pm 0,59$ \\
\hline
\end{tabular}

médio de gestação (150,64 d) e a prolificidade média (1,77 fetos) observados neste estudo foram semelhantes aos relatados para a espécie caprina por Gordon (1997). De acordo com os dados apresentados na Tabela 3, fêmeas nulíparas apresentaram menor prolificidade ( 1,41 fetos) que cabras lactantes 


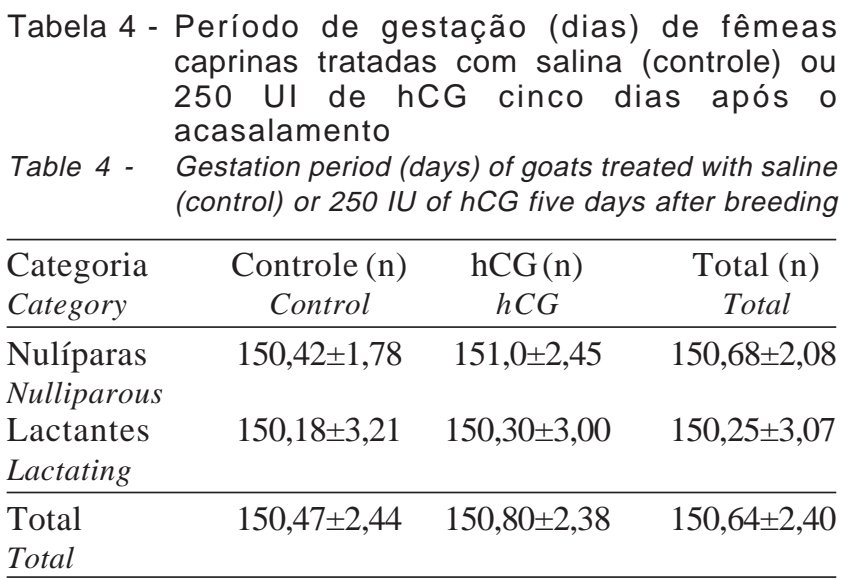

(1,90 fetos), o que é bastante comum em caprinos (Amoah \& Gelaye, 1990). A atividade ovariana (número de ovulações) e espaço uterino, superiores em cabras pluríparas, têm sido relatados como as causas mais prováveis desse fenômeno (Gordon, 1997).

\section{Conclusões}

A administração de 250 UI de hCG cinco dias após o acasalamento não melhorou o desempenho reprodutivo em fêmeas caprinas da raça Alpina. Outros estudos relativos à dosagem e do dia da administração hormonal devem ser realizados.

\section{Agradecimento}

Aos Laboratórios Calier do Brasil, pela doação do produto Vetecor ${ }^{\circledR}$, e ao $\mathrm{CNPq}$ e à FAPEMIG, pelo suporte financeiro.

\section{Literatura Citada}

AMOAH, E.A.; GELAYE, S. Reproductive performance of female goats in south pacific countries. Small Ruminant Research, v.3, p.257-267, 1990

AYRES, M.; AYRES JR., M.O.; AYRES, D.L. et al. BioEstat 2.0 Aplicações estatísticas nas áreas das ciências biológicas e médicas. Belém: Sociedade Civil Mamirauá, 2000. 272p.

CASTRO, T.; RUBIANES, E.; MENCHACA, A. et al. Ovarian dynamics, serum estradiol and progesterone concentrations during the interovulatory interval in goats. Theriogenology, v.52, p.399-411, 1999.

ENGELAND, I.V.; WALDELAND, H.; ANDRESEN, È. et al. Foetal loss in dairy goats: An epidemiological study in 22 herds. Small Ruminant Research, v.30, p.37-48, 1998.

ENGELAND, I.V.; ROPSTAD, E.; ANDRESEN, O. et al. Pregnancy diagnosis in dairy goats using progesterone assay kits and oestrous observation. Animal Reproduction Science, v.47, p.237-243, 1997.

FONSECA, J.F.; SIVA FILHO, J.M.; PINTO NETO, A. et al. Concentração plasmática de progesterona em novilhas receptoras submetidas à administração de $\mathrm{rbST}, \mathrm{GnRH}$ ou hCG no quinto dia do ciclo estral. Arquivo Brasileiro de Veterinária e Zootecnia, v.53, n.4, p.451-458, 2001a.

FONSECA, J.F.; SIVA FILHO, J.M.; PINTO NETO, A. et al. Taxas de gestação em novilhas receptoras submetidas à administração de rbST, GnRH ou hCG no quinto dia do ciclo estral. Arquivo Brasileiro de Veterinária e Zootecnia, v.53, n.4, p.459-464, 2001b.

GINTHER, O.J.; KOT, K. Follicular dynamics during the ovulatory season in goats. Theriogenology, v.42, p.987$1001,1994$.

GORDON, I. Controlled reproduction in sheep and goats. Cambridge, UK: University Press, p.62, 1997.

GREVE, T.; LEHN JENSEN, H. The effect of hCG administration on pregnancy rates following non surgical transfer of viable bovine embryos. Theriogenology, v.17, n.1, p.91, 1982. (abstr.)

ISHIDA, N.; OKADA, M.; SEBATA, K. et al. Effects of GnRH and hCG treatments for enhancing corpus luteum function to increase lambing rate of ewes artificially inseminated during the non-breeding season. Journal of Reproduction and Development, v.45, n.1, p.73-79, 1999.

KITTOK, R.J.; STELLFLUG, J.N.; LOWRY, S.R. Enhanced progesterone and pregnancy rate after gonadotropin administration in lactating ewes. Journal of Animal Science, v.56, n.3, p.652-655, 1983 .

JABLONKA-SHARIFF, A.; GRAZUL-BILSKA, A.T.; REDMER, D.A. et al. Growth and cellular proliferation of ovine corpora lutea throughout the estrous cycle. Endocrinology, v.133, p.1871, 1993.

MANN, G.E.; LAMMING, G.E.; FRAY, M.D. Plasma oestradiol and progesterone during early pregnancy in the cow and the effects of treatment with buserelin. Animal Reproduction Science, v.37, p.121-131, 1995.

MEITES, J.; WEBSTER, H.D.; YOUNG, F.W. et al. Effects of corpora lutea removal and replacement with progesterone on pregnancy in goats. Journal of Animal Science, v.10, n.2, p.411-416, 1951.

NEPHEW, K.P.; CÁRDENAS, H.; McCLURE, K.E. et al. Effects of administration of human chorionic gonadotropin or progesterone before maternal recognition of pregnancy on blastocyst development and pregnancy in sheep. Journal of Animal Science, v.72, p.453-458, 1994.

RAJAMAHENDRAN, R.; SIANANGAMA, P.C. Effect of human chorionic gonadotrophin on dominant follicles in cows: formation of accessory corpora lutea, progesterone production and pregnancy rates. Journal of Reproduction and Fertility, v.95, p.577-584, 1992.

RIBEIRO JR., J.I. Análises estatísticas no SAEG. Viçosa, MG: Universidade Federal de Viçosa, 2001. 301p.

SANGHA, G.K.; SHARMA, R.K.; GURAYA, S.S. Biology of corpus luteum in small ruminants. Small Ruminant Research, v.43, p.53-64, 2002.

SCHMITT, É.J.P.; DIAZ, T.; BARROS, C.M., de la SOTA, R.L. et al. Differential response of luteal phase and fertility in cattle following ovulation of the first-wave follicle with human chorionic gonadotropin or an agonist of gonadotropinreleasing hormone. Journal of Animal Science, v.74, p.1074-1083, 1996. 
SIANANGAMA, P.C.; RAJAMAHENDRAN, R. Effect of human chorionic gonadotropin administered at specific times following breeding on milk progesterone and pregnancy in cows. Theriogenology, v.38, p.85-96, 1992.

STAPLES, R.E.; HANSEL, W.W. Luteal function and embryo survival in the bovine. Journal of Dairy Science, v.44, p.2040, 1961. (abstr.)

THATCHER, W.W.; MOREIRA, F.; SANTOS, J.E.P. et al. Effects of hormonal treatments on reproductive performance and embryo production. Theriogenology, v.55, p.75-89, 2001.

THATCHER, W.W.; STAPLES, C.R.; DANET-DESNOYERS, G. et al. Embryo health and mortality in sheep and cattle. Journal of Animal Science, Supplement 3, v.72, p.16-30, 1994a.

THATCHER, W.W.; MACMILLAN, K.L.; HANSEN, P.J. et al. Embryonic losses: cause and prevention. In: FIELDS, M.J.; SAND, R.S. (Eds.) Factors affecting calf crop. Boca Ranton: CRC Press, 1994b. p.135-154.
WILMUT, I.; SALES, D.I.; ASHWORTH, C.J. Maternal and embryonic factors associated with prenatal loss in mammals. Journal of Reproduction and Fertility, v.76, p.851-864, 1986.

WOLFENSON, D.; ROTH, Z.; MEIDAN, R. Impaired reproduction in heat-stressed cattle: basic and applied aspects. Animal Reproduction Science, v.60-61, p.535-547, 2000.

Recebido em: 18/12/03

Aceito em: 23/02/05 